\title{
Increased Norfloxacin Skin Permeability for Fatty Alcohol Propylene Glycol (FAPG) Ointment by Optimized Process of Preparation: Behavior of Stearic Acid in Stratum Corneum Lipids
}

\author{
Hung-Hong LIN, ${ }^{a}$ Li-Ren Hsu, ${ }^{b}$ Pao-Chu Wu,${ }^{a}$ and Yi-Hung TsaI ${ }^{*, a}$ \\ School of Pharmacy, Kaohsiumg Medical College, ${ }^{a}$ Department of Pharmacy, Chia-Nan College of Pharmacy, ${ }^{b}$ \\ Taiwan, Republic of China. Received May 15, 1995; accepted July 12, 1995
}

\begin{abstract}
Preparation of the fatty alcohol propylene glycol (FAPG) ointment base plays an important role in controlling the physicochemical properties of ointments. This essay investigates the effects of preparative conditions such as cooling rate and stirring rate on the percutaneous absorption of norfloxacin from FAPG ointment. The influence of process-induced variation in enhancing effect of stearic acid which was incorporated into FAPG base was evaluated in vitro on rat skin. In the permeation experiment, norfloxacin penetration significantly increased with faster cooling rate. This result directly related to the increasing norfloxacin skin-vehicle partition coefficient. Histological analysis results showed no appreciable exfoliation of the stratum corneum. The differential scanning calorimetry (DSC) results show that stearic acid enriched lipid in the stratum corneum resulting from treatment with supercooling products may result in more crystalline structure and, hence, preferential partitioning of the norfloxacin into the more crystalline regions of the membrane can be observed. A much greater enhancing effect can be achieved when we use stearic acid together with norfloxacin in propylene glycol (PG); but such effect cannot be found if 5 wt $\%$ stearic acid/PG suspension is used to pretreat skin before the application of norfloxacin PG solution.
\end{abstract}

Key words norfloxacin; fatty alcohol propylene glycol ointment; preparative condition; stearic acid; stratum corneum; differential scanning calorimetry (DSC)

For transdermal preparations, the stratum corneum layer is known to be a major barrier to drug permeation in the keratinized epithelia. Owing to the poor skin penetration of drug, topical administration is often limited and is little effective. ${ }^{1)}$ Since many drugs cannot permeate the skin in the amount necessary to initiate therapeutic effects, transport can be facilitated by employing penetration enhancers. From the viewpoint of safety, it is desirable that enhancers be materials conventionally used in pharmaceutical preparations. Various fatty acids have been examined as enhancers for percutaneous absorption. $^{2-4)}$

In previous studies, stearic acid was chosen to incorporate into a fatty alcohol propylene glycol (FAPG) base to promote the absorption of quinolone antibiotics from ointment and to improve the storage properties. ${ }^{5)}$ It has been found that stearic acid is an effective enhancer but poorly soluble in propylene glycol (PG). As stated before, ${ }^{6}$ stearic acid is polymorphic and can exist in more than one crystalline form. The polymorphic modifications are strongly affected by both solvents and preparative conditions.

Like FAPG ointments, when stearic acid combines with stearyl alcohol, the supersaturation of the acid and precipitated forms in PG no longer exist because stearic acid easily dissolves in the melting stearyl alcohol. ${ }^{7)}$ On the other hand, if the manufacturing process were changed, the physical properties of the ointment would be remarkably different. ${ }^{8)}$ Stearic acid might be susceptible to the process-induced variation in its final enhancing effect. Although there are reports of the effects of several penetration enhancers, relative comparison of the understood effects of a single enhancer within various manufacturing processes, as we have done, are much less frequent. Moreover, no mechanism by which stearic acid facilitates

* To whom correspondence should be addressed. the skin penetration of norfloxacin has been reported. To optimize absorption, a study of appropriate preparation conditions is necessary.

In the present study, we selected norfloxacin as a model drug. It is a second-generation quinolone and usually commonly used in the treatment of urinary tract infections, gastrointestinal and other infections. ${ }^{9)}$ We have now investigated the influence of process-induced variation on in vitro percutaneous absorption of norfloxacin from an FAPG base. Furthermore, the relationship between the penetration enhancing effect of stearic acid and the preparation conditions of ointment are also discussed. In addition, complementary differential scanning calorimetry (DSC) was employed in an attempt to probe potential alterations in the keratin structure.

\section{MATERIALS AND METHODS}

Materials Stearic acid, PG and urethane were purchased from Sigma Chemical Co.; stearyl alcohol and polyethylene glycol 6000 were purchased from E. Merck Co.; 1,2,6-hexanetriol was purchased from Riedel de. Haen Co.; all other chemicals were of analytical grade.

Skin Preparation Rat skins were harvested from male Wistar rats weighing $230-270 \mathrm{~g}$. After being killed in a $\mathrm{CO}_{2}$ chamber, the abdominal hair was removed with electric clippers. A $3 \times 3 \mathrm{~cm}^{2}$ section of denuded skin with a thickness of $0.65-0.75 \mathrm{~mm}$ was excised immediately before the permeation experiment.

Preparation of FAPG Ointments The FAPG base is composed of stearyl alcohol $(23.5 \% \mathrm{w} / \mathrm{w})$, PG $(61.9 \%)$, polyethylene glycol $6000(4.8 \%), 1,2,6$-hexanetriol $(4.7 \%)$ and stearic acid. Stearic acid, an absorption enhancer, was incorporated at a concentration of $5 \%$. Norfloxacin was dissolved in each bases at a concentration of $0.1 \%$. The ointments were prepared with the reported method ${ }^{10}$ (C) 1995 Pharmaceutical Society of Japan 
after slight modification. The preparative conditions of ointment such as stirring rate and cooling rate were selected as independent variables. The so-called "supercooling operation" or "slow cooling operation" was carried out and kept in a thermostatic ice bath (Model PC-36B, Julabo Instruments, Ltd., Germany). The range of cooling rate from 0.115 to $0.769\left({ }^{\circ} \mathrm{C} / \mathrm{s}\right)$ was accompanied by a stirring rate of 200 or $600 \mathrm{rpm}$. The process was described in more detail in an earlier study. ${ }^{11)}$ In order to gain further insight into the role stearic acid plays in norfloxacin penetration through rat skin, the stearic acid/PG suspensions containing $0.1 \mathrm{wt} \%$ norfloxacin were prepared by crystallizing stearic acid from PG in the presence of $5 \mathrm{wt} \%$ 1,2,6-hexanetriol. Cooling operations were the same as stated above.

Wet Dressing Method for Pretreatment The method for pretreatment was described previously ${ }^{12)}$ and was used with some modifications. The rats were anesthetized with urethane $(1 \mathrm{~g} / \mathrm{kg}$, i.p./q $24 \mathrm{~h})$ and fixed on a plate during the period of pretreatment with penetration enhancer. The abdominal hair was removed by clippers. A piece of cotton cloth, $3 \times 5 \mathrm{~cm}^{2}$, was dampened with $5 \mathrm{wt} \%$ stearic acid/ PG suspension or PG free of stearic acid as a control. Then, the damp cloth was applied to the shaved surface of the rat for $48 \mathrm{~h}$ by the occlusive dressing technique (ODT) ${ }^{13)}$ Subsequently, the wet dressing was peeled off and the applied area was gently swabbed clean several times with cotton to remove the residual solution without damaging the skin. The skin was excised for the permeation experiment.

Determination of Permeation through Rat Skin The diffusion cells were used in a way similar to the apparatus of the Franz diffusion assembly. ${ }^{14)}$ The excised rat skin was used as the membrane for skin permeation experiments. The skin was positioned with the stratum corneum facing the donor cell, and the dermis side was in contact with the receiver compartment which was filled with phosphate buffer ( $\mathrm{pH} 7.4$, containing $20 \% \mathrm{w} / \mathrm{w}$ PEG 400). The area available for diffusion was $2.43 \mathrm{~cm}^{2}$; either $2.0 \mathrm{~g}$ of ointment or the $0.1 \mathrm{wt} \%$ norfloxacin PG solution (with or without stearic acid) was put into the donor cell. The receiver compartment was agitated by a magnetic stirrer at $700 \mathrm{rpm}$. The apparatus was maintained at $37 \pm 0.5^{\circ} \mathrm{C}$ with a water jacket. An aliquot $(0.5 \mathrm{ml})$ of the sample was taken from the receiver compartment at appropriate times, and the concentration of norfloxacin was determined by HPLC method. After each sampling, the same volume of fresh phosphate buffer was added to the receiver compartment to keep the volume constant.

Measurement of Apparent Partition Coefficient The degree of norfloxacin partitioning between isopropyl palmitate (oil phase) and PG (water phase) was determined as follows: stearic acid/PG suspension $5 \mathrm{~g}$ containing $0.1 \mathrm{wt} \%$ norfloxacin and isopropyl palmitate $5 \mathrm{ml}$ were placed in a glass-stoppered test tube and shaken in a water bath at $37^{\circ} \mathrm{C}$ for $2 \mathrm{~d}$. The mixture was centrifuged at $3000 \mathrm{rpm}$ for $10 \mathrm{~min}$. The PG layer was filtered through a syringe filter with pore size $0.45 \mu \mathrm{m}$ (Msi. Micro Separation Inc.) and the concentration of norfloxacin in PG was determined by HPLC.

Analytical Methods The amount of norfloxacin in each sample was determined by HPLC. Pipemidic acid was used as an internal standard. The conditions were as follows: pump, model L-6000 (Hitachi); column, 4.6× $250 \mathrm{~mm}$ Spheris C18 (Phase Separations Ltd., U.K.); mobile phase, $0.015 \mathrm{M}$ tetrabutyl ammonium iodide solution-acetonitrile $(95: 5, \mathrm{v} / \mathrm{v})$; UV detector, model L-4000 (Hitachi); wavelength, $275 \mathrm{~nm}$; flow rate, $1 \mathrm{ml} / \mathrm{min}$. Peak areas were calculated by using a chromato-integrator, model D-2500 (Hitachi).

Analyses of Data The in vitro penetration parameters were calculated from the penetration data using the following equations ${ }^{15)}$ :

$$
\begin{aligned}
& J_{\mathrm{ss}}=K_{\mathrm{p}} C_{\mathrm{s}} \\
& K_{\mathrm{p}}=K_{\mathrm{m}} D / h \\
& T_{\mathrm{L}}=h^{2} / 6 D
\end{aligned}
$$

where $J_{\mathrm{ss}}$ and $C_{\mathrm{s}}$ are the steady-state flux and donor concentration respectively, $K_{\mathrm{m}}$ is the effective skin-vehicle drug partition coefficient, $D$ is the diffusion constant within the skin, $K_{\mathrm{p}}$ is the permeability coefficient through the skin, $T_{\mathrm{L}}$ is the lag time, and $h$ is the effective barrier thickness.

Histological Examination of the Stratum Corneum Histological changes in the stratum corneum were examined after the permeation experiments. The skin was fixed in formalin by the conventional procedure, stained with hematoxylin-eosin and examined under a microscope. Pretreated skin with PG free of stearic acid served as a control.

Stratum Corneum Preparation Stratum corneum sheets were prepared from rat skin by trypsin treatment after the permeation experiment. The epidermis was separated from underlying layers after several hours of incubation on filter paper saturated with $0.5 \%$ crude trypsin (Type II-Sigma) in phosphate buffered saline at $37^{\circ} \mathrm{C}$. This sample was washed with distilled water and. spread on wire mesh to dry. Samples were stored in a desiccator until use.

DSC Prior to DSC, all samples were placed for several days in a chamber maintained at $95 \%$ relative humidity. Stratum corneum sheets were equilibrated to a water content of about $30 \%(\mathrm{w} / \mathrm{w})$ under the above conditions. The differential scanning calorimeter (Perkin Elmer DSC7) was used at a scan rate of $5^{\circ} \mathrm{C} / \mathrm{min}$. The temperature range was from 25 to $160^{\circ} \mathrm{C}$.

\section{RESULTS AND DISCUSSION}

Norfloxacin penetration amount-time profiles from the in vitro skin permeation experiments are shown in Fig. 1. A linear regression analysis was used to determine the steady-state flux $\left(J_{\mathrm{ss}}\right)$ of drug for each sample. The permeability coefficients, $K_{\mathrm{p}}\left(\mathrm{cm} \cdot \mathrm{h}^{-1}\right)$, are given by Eq. 1 . The diffusion coefficient $\left(D_{\mathrm{m}}\right)$ of drug through the skin can be estimated from the lag time according to Eq. 2. Thus the skin/vehicle partition coefficient $\left(K_{\mathrm{m}}\right)$ can be calculated indirectly by Eq. 3 . The kinetic parameters calculated from these penetration profiles are listed in Table 1 . It can be seen that norfloxacin flux significantly increased with faster cooling rates $(p<0.05)$ at a constant stirring rate of $600 \mathrm{rpm}$. Moreover, the slower of the two 
Table 1. Effect of Various Preparation Conditions on Percutaneous Penetration Parameters of Norfloxacin through Rat Skin

\begin{tabular}{|c|c|c|c|c|c|c|}
\hline \multicolumn{2}{|c|}{ Preparation conditions } & \multicolumn{5}{|c|}{ Penetration parameters } \\
\hline No. & $\begin{array}{l}\text { Cooling rate } \\
\left({ }^{\circ} \mathrm{C} / \mathrm{s}\right)\end{array}$ & $\begin{array}{c}J_{\mathrm{ss}} \\
\left(\mu \mathrm{g} / \mathrm{cm}^{2} \mathrm{~h}\right)\end{array}$ & $\begin{array}{l}D \times 10^{-4} \\
\left(\mathrm{~cm}^{2} / \mathrm{h}\right)\end{array}$ & $K_{\mathrm{m}} \times 10^{-1}$ & $\begin{array}{l}K_{\mathrm{p}} \times 10^{-3} \\
(\mathrm{~cm} / \mathrm{h})\end{array}$ & $\begin{array}{l}T_{\mathbf{L}} \\
(\mathrm{h})\end{array}$ \\
\hline 1. & $0.115 \pm 0.007^{a)}$ & $1.51 \pm 0.02$ & $3.17 \pm 0.24$ & $3.35 \pm 0.29$ & $1.51 \pm 0.02$ & $2.59 \pm 0.19$ \\
\hline 2. & $0.442 \pm 0.002^{a)}$ & $2.00 \pm 0.30^{d)}$ & $3.27 \pm 0.18$ & $4.25+0.54$ & $2.00+0.30^{d)}$ & $2.50+0.14$ \\
\hline 3. & $0.769 \pm 0.002^{a)}$ & $2.86 \pm 0.15^{c)}$ & $3.44 \pm 0.31$ & $5.86 \pm 0.61^{c)}$ & $2.86 \pm 0.15^{c}$ & $2.40+0.23$ \\
\hline 4. & $0.441 \pm 0.001^{b)}$ & $2.38 \pm 0.18^{c)}$ & $3.30 \pm 0.17$ & $5.07 \pm 0.57$ & $2.38 \pm 0.18^{c)}$ & $2.48 \pm 0.13$ \\
\hline
\end{tabular}

Each value represents the mean \pm S.D. $(n=3)$. a) Stirring rate, $600 \mathrm{rpm} . \quad$ b) Stirring rate, $200 \mathrm{rpm} . \quad$ c) Significantly different at an alpha level of 0.05 from that of No. 1. d) Significantly different at an alpha level of 0.05 between No. 2 and No. 3 .

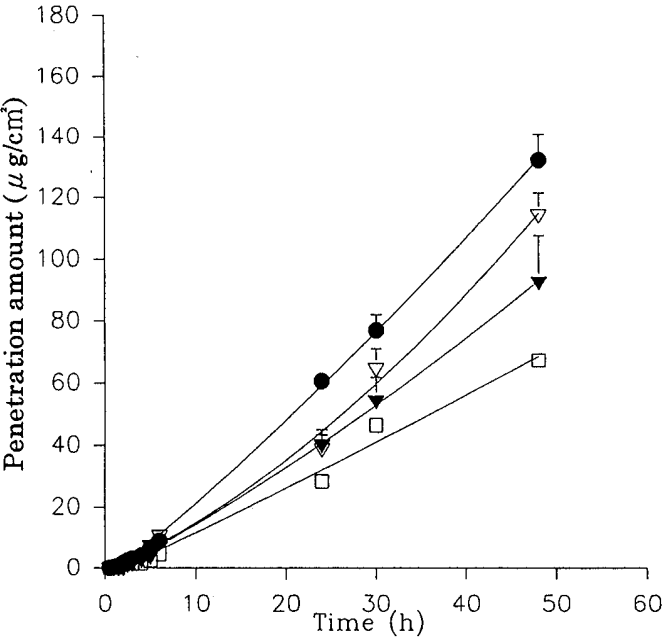

Fig. 1. Effect of Various Preparative Conditions on the Percutaneous Absorption of Norfloxacin through Rat Skin

$\square$, condition No. $1 ; \boldsymbol{\nabla}$, No. 2 ;, No. $3 ; \nabla$, No. 4. Points and vertical bars represent the mean \pm S.D. $(n=3)$, respectively.

stirring rates of equal cooling rate gave higher $J_{\text {ss }}$ value, but there was no significant difference between them. Correlative to our previous release study ${ }^{11}$ on those results, the transdermal permeation rate is much lower than the release rate of norfloxacin from the FAPG base. Briefly, the permeation of norfloxacin through the skin is the rate-determining step for percutaneous absorption of norfloxacin from FAPG ointment. Furthermore, the overall increase in norfloxacin penetration was mainly due to the increase in $K_{\mathrm{m}}$ rather than $D$.

In the present study, the $C_{\mathrm{s}}$ values of norfloxacin in PG of the FAPG bases with various manufacturing processes were assumed to be the same. The assumption may be not valid, however, because the fractional concentration of norfloxacin varied in the different concentrations of stearic acid in PG. As stated, ${ }^{7)}$ the content of stearic acid in PG is susceptible to process-induced variation, hence the drug activities are not the same. Based on these results, stearic $\mathrm{acid} / \mathrm{PG}$ suspension was selected for further evaluation of the enhancing effect.

Figure 2 presents the penetration profiles of norfloxacin through excised rat skins which were pretreated with $P G$ alone or pretreated with stearic acid/PG suspension; the rat skins were then subjected to the same penetration experiment using $0.1 \mathrm{wt} \%$ norfloxacin PG suspension containing $5 \mathrm{wt} \%$ or free of stearic acid, respectively. The penetration parameters calculated from each penetration

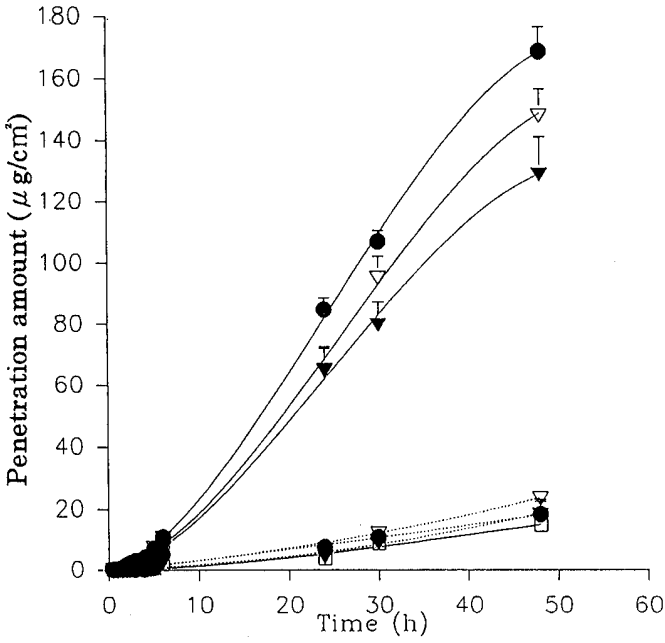

Fig. 2. Effect of Potentation of Stearic Acid on the Percutaneous Absorption of Norfloxacin through Rat Skin

Reapplication of $0.1 \%$ norfloxacin PG containing stearic acid on pretreated skin with PG: $-\nabla-$, condition No. $1 ;-\nabla-$, No. $2 ;-1-$, No. $3 ;-\square-$, without stearic acid. Reapplication of $0.1 \%$ norfloxacin PG free of stearic acid on pretreated skin with stearic acid/PG suspension; $\cdots \nabla \cdots$, condition No. $1 ; \cdots \nabla \cdots$, No. 2 ; ..., No. 3 .

profile are listed in Table 2. It can be seen that the flux and the skin-vehicle drug partition coefficient of the former treatment was significantly larger than the latter. Increasing the cooling rate also resulted in greater enhancement of norfloxacin skin penetration because of higher stearic acid concentration in PG. However, the diffusion coefficient of norfloxacin did not significantly increase with the increase in amount of stearic acid in PG owing to preparation by supercooling (data not shown). In accord with the studies previously reported, ${ }^{16,17)}$ at least the following assumptions can be made as to the mechanisms of the enhancing effect of fatty acids: they interact with some components in the stratum corneum, they cause an increase in partition coefficient, or they have a fluidizing effects on the stratum corneum. To examine whether an increase in norfloxacin penetration was directly related with skin-vehicle partition coefficient $\left(K_{\mathrm{m}}\right)$, the effect of stearic acid on norfloxacin partition between isopropyl palmitate and PG containing stearic acid was determined. The calculated skin-vehicle partition coefficients were found to agree well with the partition coefficient of isopropyl palmitate/PG-stearic acid suspension. This might be the reason that pretreating the skin with stearic acid/PG suspension before the application of $0.1 \mathrm{wt} \%$ norfloxacin $\mathrm{PG}$ solution showed only minor 
Table 2. Effect of Preparation Condition on Partition Coefficient and Percutaneous Penetration Parameters of Norfloxacin through Rat Skin

\begin{tabular}{|c|c|c|c|c|c|c|}
\hline \multicolumn{2}{|c|}{ Preparation conditions } & \multirow{2}{*}{$\mathrm{Pc}^{b)}$} & \multicolumn{2}{|c|}{ Skin pretreated with $\mathrm{PG}^{c)}$} & \multicolumn{2}{|c|}{ Skin pretreated with stearic acid/PG ${ }^{d)}$} \\
\hline No. & $\begin{array}{c}\text { Cooling rate }{ }^{a)} \\
\left({ }^{\circ} \mathrm{C} / \mathrm{s}\right)\end{array}$ & & $\begin{array}{c}J_{\mathrm{ss}} \\
\left(\mu \mathrm{g} / \mathrm{cm}^{2} \mathrm{~h}\right)\end{array}$ & $K_{\mathrm{m}} \times 10^{-1}$ & $\begin{array}{c}J_{\mathrm{ss}} \\
\left(\mu \mathrm{g} / \mathrm{cm}^{2} \mathrm{~h}\right)\end{array}$ & $K_{\mathrm{m}} \times 10^{-1}$ \\
\hline 1. & $0.115 \pm 0.007$ & $0.28 \pm 0.02$ & $3.05 \pm 0.04$ & $6.32 \pm 0.08$ & $0.37 \pm 0.02$ & $1.85 \pm 0.47$ \\
\hline 2. & $0.442 \pm 0.002$ & $0.31 \pm 0.03$ & $3.25 \pm 0.20$ & $6.71 \pm 0.21$ & $0.49 \pm 0.07$ & $2.80 \pm 0.93$ \\
\hline 3. & $0.769 \pm 0.002$ & $0.39 \pm 0.03^{e)}$ & $3.74 \pm 0.15^{e)}$ & $7.02 \pm 0.07^{e)}$ & $0.41 \pm 0.09$ & $2.40 \pm 0.23$ \\
\hline
\end{tabular}

Each value represents the mean \pm S.D. $(n=3)$. a) Stirring rate, $600 \mathrm{rpm}$. b) Partition coefficient of isopropyl palmitate/PG suspension. $c$ ) Reapplication of $0.1 \%$ norfloxacin PG containing stearic acid on skin pretreated with PG. d) Reapplication of $0.1 \%$ norfloxacin PG free of stearic acid on skin pretreated with stearic acid/PG suspension. e) Significant different at an alpha level from that of No. 1.

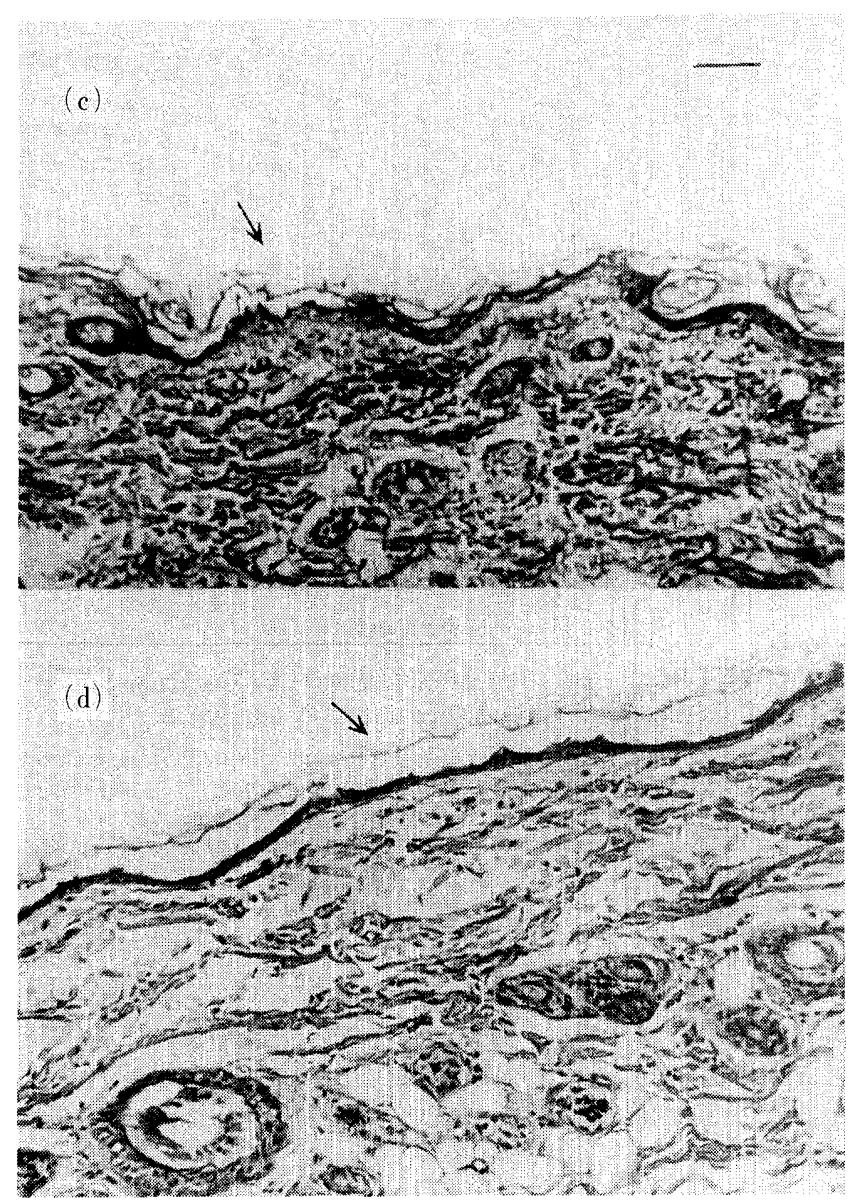

Fig. 3. Stained Cross Section of Stratum Corneum of Rat Skin

Arrows indicate the stratum corneum. (a) Skin treated with PG as control, (b) skin pretreated with 5\% stearic acid/PG suspension, (c) application of $0.1 \%$ norfloxacin PG together with stearic acid, (d) application of FAPG ointment. Bar $=5 \mu \mathrm{m}$.

enhancement, but a much greater effect was achieved when stearic acid was used together with norfloxacin in PG.

The histological analysis of the cross-sections of abdominal skin were randomly selected from each permeation experiment and the photomicrograph is shown in Fig. 3. The skin pretreated with $5 \mathrm{wt} \%$ stearic acid/PG suspension did not undergo appreciable exfoliation of the stratum corneum (Fig. 3b). Similar results can be seen in the application of $0.1 \mathrm{wt} \%$ norfloxacin PG together with stearic acid (Fig. 3c), or FAPG ointments (Fig. 3d). Although the epithelium tissue appears slightly more swollen than the control (Fig. 3a), the stratum corneum remains undisturbed.
In the former, histological examination of the stratum corneum indicated that the treatment with stearic acid did not cause significant changes or appreciable damage to the stratum corneum. To elucidate the influence of preparation conditions on the mechanisms for the enhancing effect of stearic acid and pathways for the diffusion of drugs in the stratum corneum, differential thermal analysis was utilized to establish structure-property relationship at the molecular and macromolecular level.

Figure $4 \mathrm{~A}$ shows the DSC thermal transition pattern obtained following treatment of the rat stratum corneum with PG alone. This thermal profile is similar to that reported previously by Wilkes et al. ${ }^{18)}$ Two major transitions are observed occurring at $65-70^{\circ} \mathrm{C}$ and $95-$ 


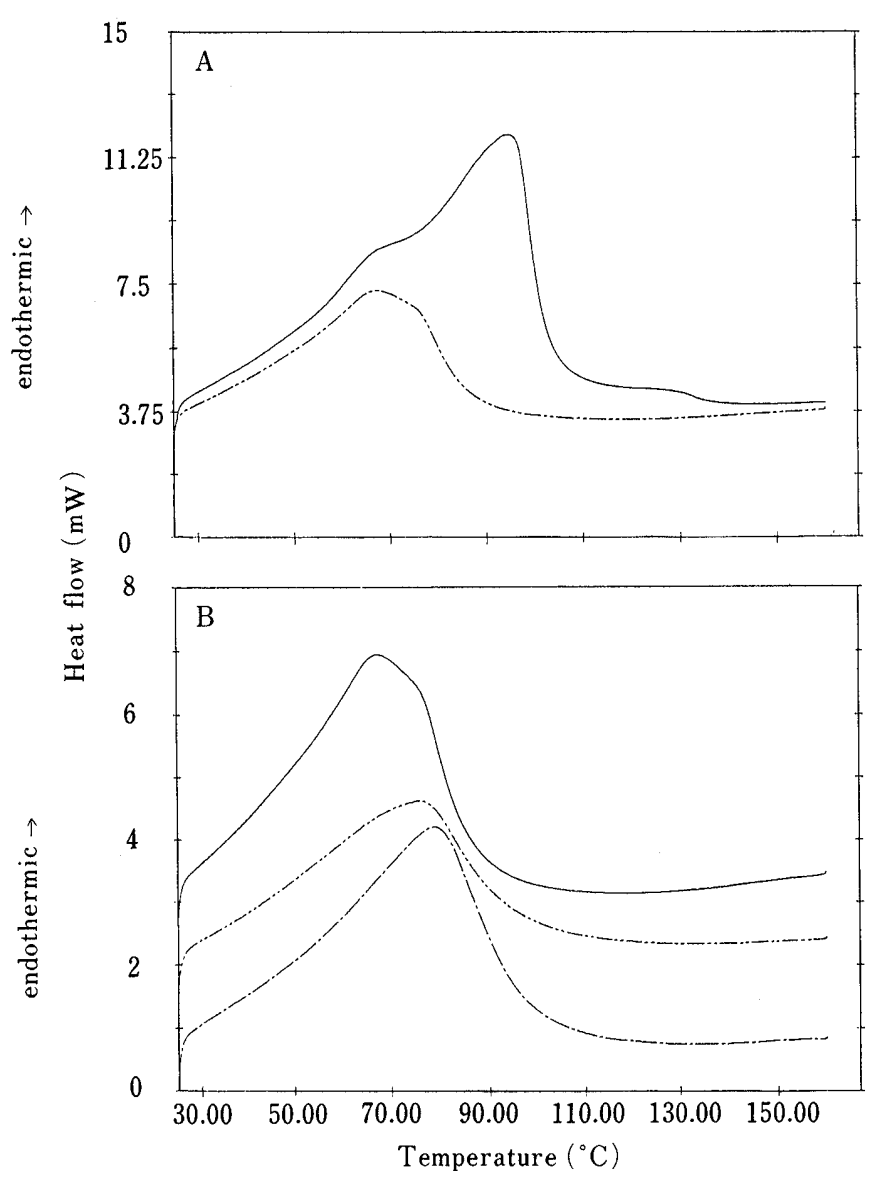

Fig. 4. The DSC Thermal Profile of Rat Stratum Corneum Treated with FAPG Ointment by Various Preparative Conditions

A. - treated with PG as control; - - - , treated with FAPG ointment by conventional preparation. B. - - by conventional preparation; -..., by fastercooling; $-\ldots-\ldots$, by supercooling. The cooling rates; conventional products $\left(0.115^{\circ} \mathrm{C} / \mathrm{s}\right)$; faster-cooling products $\left(0.442^{\circ} \mathrm{C} / \mathrm{s}\right)$; supercooling products $\left(0.769^{\circ} \mathrm{C} /\right.$

$100^{\circ} \mathrm{C}$. When compared to this reference, these two peaks are probably due to a transition of intercellular lipids and lipid-protein complexes, respectively. But the transition normally occurring at $40^{\circ} \mathrm{C}$ attributed to surface sebaceous lipids is too weak to detect. The lower trace represents the thermal profile of the stratum corneum $24 \mathrm{~h}$ after application with FAPG ointment by conventional preparation and shows no thermal phase transition other than the lipid transition. The absence of a lipid-protein transition indicates that certain exogenously applied stearic acid may disrupt the stratum corneum lipid-protein structure. Simultaneously, the stratum corneum lipids contain a large amount of free and esterified long chain fatty acids, ${ }^{19)}$ so the stearic acid containing 18 carbon atoms length should partition into this domain. As has been demonstrated by Chester et al. ${ }^{20)}$ stearic acid enrichment of cell membrane results in a more crystalline structure, whereas unsaturated fatty acid enrichment results in a more fluid lipid bilayer as compared with control. Furthermore, the trend toward increased crystalline lipid content might exhibit a dramatically altered thermal phase transition. ${ }^{18)}$ As seen in Fig. 4B, the main endotherm of the stearic acid enriched lipids resulting from treatment with supercooling products is shifted markedly towards higher temperatures in comparison with treatment by faster co- oling or conventional products. Furthermore, the thermal profiles of stratum corneum pretreatment with stearic acid/PG suspension are also similar to the above results.

An earlier study by Copper ${ }^{21)}$ showed that there are at least two pathways for transport of materials across the skin: a polar pathway and a nonpolar pathway. The polar pathway is associated with the protein component of the stratum corneum and might be envisioned as aqueous channels in the protein; the nonpolar pathway is associated with the lipid component. If the protein thermal transition temperature is affected, there must be an interaction with the protein. Because norfloxacin is a lipophilic molecule and stearic acid is sparingly soluble in PG, the effect of stearic acid is reduced on the polar pathway. In contrast, the continuous lipid pathway is believed to account for the transport of lipophilic molecules. Moreover, the in vitro flux of norfloxacin significantly increased with the faster cooling rate which resulted in a more crystalline structure of the stratum corneum. Thus, preferential partitioning of the norfloxacin into the more crystalline regions of the membrane can be observed while using stearic acid together with norfloxacin in PG, consistent with the reconstituted transporter/model membrane system. ${ }^{22)}$

\section{CONCLUSIONS}

While the use of stearic acid as penetration enhancer has been previously reported, the present investigation is the first to probe the effect of preparation condition of topical products on drug skin permeability and to propose a molecular mechanism by which stearic acid might act. It can be assumed that the permeation of norfloxacin through the skin is the rate-determining step for percutaneous absorption of norfloxacin from FAPG ointment. The enhancement of skin permeability of norfloxacin with stearic acid can be imporveed by increasing the cooling rate of the manufacturing process; the main reason is the increase in norfloxacin partitioning between the skin and the vehicle. Furthermore, surpercooling induced maximum norfloxacin permeation and showed an approximately 1.9 -fold greater permeation flux for norfloxacin penetration. The results of DSC experiments on the stratum corneum indicate that stearic acid enriched lipids resulting from treatment with supercooling products may result in a more crystalline structure and, hence, preferential partitioning of the norfloxacin into the more crystalline regions of the membrane can be observed. The side effect of removal of the epithelial barrier by some enhancers was not found in the histological analysis of incorporation of stearic acid into FAPG ointment. It is possible to control the processing of FAPG ointments to predict and obtain a reproducible product with desired physical properties as well as optimizing the penetration enhancement without skin irritation.

Acknowledgment The work was partly supported by a grant from Cheng's Pharmaceutical Sciences Foundation, Taipei, Taiwan, Republic of China. 


\section{REFERENCES}

1) Udupa N., Bhat L., Drug Dev. Ind. Pharm., 18, 2197 (1992).

2) Aungst B. J., Rogers N. J., Shefter E., Int. J. Pharmaceut., 33, 225 (1986).

3) Yamada M., Uda Y., Chem. Pharm. Bull., 35, 3390 (1987).

4) Komata Y., Inaoka M., Kaneko A., Fujie T., J. Pharm. Sci., 81, 744 (1992).

5) Jaw Y. I., Master Thesis, Kaohsiung Medical College, 1991.

6) Gart N., Wellnen E., Saring S., Journal of Crystal Growth, 57, 577 (1982).

7) Lin H. H., Huang Y. B., Hsu L. R., Tsai Y. H., Int. J. Pharmaceut., 112, 165 (1994).

8) Timmins P., Browning I., Payne N. I., J. Pharm. Pharmacol., 42, 583 (1990).

9) Holmes H., Brogden R. N., Richards D. M., Drugs, 30, 482 (1985).

10) Kaiho F., Nomura H., Makabe E., Kato Y., Chem. Pharm. Bull., 35, 2928 (1987).
11) Lin H. H., Hsu L. R., Huang Y. B., Tsai Y. H., Chin. Pharm. J., 46, 73 (1994).

12) Hsu L. R., Tsai Y. H., Huang Y. B., Int. J. Pharmaceut., 71, 193 (1991).

13) Natio S. I., Tsai Y. H., Int. J. Pharmaceut., 8, 263 (1981).

14) Chien Y. W., Drug Dev. Ind. Pharm., 13, 589 (1987).

15) Chow D. S.-L., Kaka I., Wang T. I., J. Pharm. Sci., 73, 1794 (1984).

16) Melchior D. L., Czech M. P., J. Biol. Chem., 254, 8744 (1979).

17) Kim C. K., Kim J. J., Chi S. C., Shim C. K., Int. J. Pharmaceut., 99, 109 (1993).

18) Wilkes G. L., Nguyen A.-L., Wildnauer R., Biochem. Biophys. Acta, 304, 267 (1973).

19) Golden G. M., Mckie J. E., Potts R. O., J. Pharm. Sci., 76, 25 (1987).

20) Chester D. W., Tourtellotte M. E., Melchior D. L., Romano A. H., Biochim. Biophys. Acta, 860, 383 (1986).

21) Cooper E. R., J. Pharm. Sci., 73, 1153 (1984).

22) Ogiso T., Shintani M., J. Pharm. Sci., 79, 1065 (1990). 